\title{
Spectroscopic distance estimates for fourteen faint red LHS and NLTT stars
}

\author{
H. Jahreiß ${ }^{1}$, R. Scholz ${ }^{2, \star}$, H. Meusinger ${ }^{3, \star}$, and I. Lehmann ${ }^{2,4, \star}$ \\ 1 Astronomisches Rechen-Institut, Mönchhofstraße 12-14, 69120 Heidelberg, Germany \\ 2 Astrophysikalisches Institut Potsdam, An der Sternwarte 16, 14482 Potsdam, Germany \\ 3 Thüringer Landessternwarte Tautenburg, 07778 Tautenburg, Germany \\ 4 Department of Astronomy \& Astrophysics, The Pennsylvania State University, 525 Davey Laboratory, \\ University Park, PA 16802-6305, USA
}

Received 29 December 2000 / Accepted 14 February 2001

\begin{abstract}
Spectroscopic observations were carried out of potential nearby high proper motion stars taken from Luyten's LHS and NLTT catalogues. The purpose was to obtain a first distance estimate for hitherto neglected nearby candidates. The resulting spectra for fourteen stars are presented and discussed. The sample comprises eleven $\mathrm{M}$ dwarfs and three $\mathrm{M}$ subdwarfs having spectroscopic distances from $17 \mathrm{pc}$ to $160 \mathrm{pc}$.
\end{abstract}

Key words. stars: distances - stars: late type - galaxy: solar neighbourhood

\section{Introduction}

Two decades ago Willem Luyten (1979-80) published the NLTT (New Luyten Catalogue of Stars with Proper Motions Larger than Two Tenths of an Arcsecond) containing all stars then known with proper motions larger than 0.180 arcsec/year. At that time, the NLTT was the most comprehensive compilation of high proper motion stars and this is still so. The faint part of this catalogue is based on Luyten's own measurements of the POSS-I plates from the Palomar National Geographic Survey which cover the area north of declination $-33^{\circ}$, i.e. 77 per cent of the entire sky. The magnitude limit of the POSS-I plates $m_{\mathrm{pg}} \sim 21^{\mathrm{m}}$ and $m_{R} \sim 18^{\mathrm{m}}$ determined also the faint limit of the NLTT. All magnitudes fainter than $m_{\mathrm{pg}}=10^{\mathrm{m}}$ were eye estimates carried out by Luyten himself by interpolating between the magnitudes of Durchmusterung stars (Argelander 1859-62; Schönfeld 1886; Thome \& Perrine 1892-1932) and the plate limit.

The optimal detection efficiency was obtained for stars having photographic magnitudes from $13^{\mathrm{m}}$ to $17^{\mathrm{m}}$. The galactic belt $|b| \leq 15^{\circ}$ had to be excluded to avoid crowding. The faintest proper motion stars south of the POSS-I limit at declination $-33^{\circ}$ are still provided by Luyten's Bruce Proper Motion Survey completed in 1937 with lim-

Send offprint requests to: H. Jahreiß, e-mail: jahreiss@ari.uni-heidelberg.de

* Visiting Astronomer, German-Spanish Astronomical Centre, Calar Alto, operated by the Max-Planck-Institute for Astronomy, Heidelberg, jointly with the Spanish National Commission for Astronomy. iting photographic magnitudes from 15.5 to 17.5 on long exposure plates.

The appearance of NLTT and LHS (with $\mu \geq$ $0.500 \mathrm{arcsec} / \mathrm{yr}$ the high proper motion part of NLTT) gave new impetus to the search for faint nearby stars in that it provided a preselection of promising nearby candidates. VRI photometry was measured by Eggen $(1979,1980,1987)$ for all LHS stars south of declination $+30^{\circ}$ and brighter than 15.1 visual magnitude. Eggen's photometric survey was completed by Weis (1996). In a series of another four papers (see e.g. Weis 1986) the same author provided VRI photometry for selected northern NLTT stars characterized as late type stars by Luyten's spectral class " $m$ ". After publication of the GJ supplement (Gliese \& Jahreiß 1979) these photometric surveys of LHS and NLTT stars constituted one of the major sources of new nearby objects that could be added to the following compilation published in the form of the preliminary CNS3 (Gliese \& Jahreiß 1991).

It should be added that more or less all faint targets of the USNO parallax programme were chosen from Luyten's catalogues, and the resulting precise USNO parallaxes allowed more reliable calibrations of photometric and spectroscopic distances. Last but not least, another success for the NLTT catalogue: many brighter NLTT stars were selected for observation by the HIPPARCOS astrometric satellite, and finally, it turned out that the nearest newly discovered nearby star Hip $103039=$ LP 816-60 was a NLTT star.

These examples demonstrate that most efforts, except perhaps the USNO parallaxes, were limited in magnitude. 
In other words, the real magnitude limit of the POSS-I plates exploited by Luyten remained mostly out of reach.

Apart from the spectral classes determined from estimated differences between red and blue plates of his survey, Willem Luyten also gave in LHS and NLTT ordinary spectral types from various sources. However, these spectral types are not suitable for reliable spectroscopic distance estimates, because their origin cannot easily be recovered or remains often unknown, and at that time, $\mathrm{K}$ and $\mathrm{M}$ dwarf spectra were published in numerous different systems each requiring its own luminosity calibration. An exhaustive compilation of such spectral types for the LHS stars can be found in Kirkpatrick (1992).

In recent years, broad wavelength spectroscopy in the range between $6000 \AA$ and $9000 \AA$ was established as a powerful tool in investigating K5 to M9 dwarf stars (Kirkpatrick 1995). From then on, spectroscopic observations, even with low resolution and under varying sky conditions, provide a fast method to obtain spectral types and first estimates on the distances of late type dwarf stars (see also Henry et al. 1994). This allows a first preselection of nearby stars before a more extensive trigonometric parallax determination is undertaken.

In addition to our new high-proper motion survey in the southern sky (Scholz et al. 1999, 2000; Schweitzer et al. 1999; Ibata et al. 2000), we have drawn attention to relatively bright $\left(R<17^{\mathrm{m}}\right)$ and red proper motion stars north of declination $-30^{\circ}$ for which no spectral types were available. Spectroscopic observations of such bright stars can be carried out within a back-up programme designed for periods of cloudy weather.

\section{Data sample and observations}

The NLTT contains altogether 12345 stars of Luyten's spectral class " $m$ " north of declination $-30^{\circ}$ and in the magnitude interval $16.8 \geq m_{R} \geq 13.5$, i.e. stars fainter than the photometric surveys described in the introduction and brighter than $R=17^{\mathrm{m}}$ (the APM measured $R$ transforms to Luyten's $m_{R}=16.8$ according to the relation given in Scholz et al. 2000). A further restriction to stars having annual proper motions larger than 0.35 arcsec leaves a total of 2192 "m"-stars which are presumably hitherto uninvestigated.

Having this in mind, we prepared a list of 31 "bad weather targets", 25 NLTT stars and 6 LHS stars. We selected NLTT stars from the appropriate sky region between $10^{\mathrm{h}}$ and $14^{\mathrm{h}}$ in right ascension and between $-20^{\circ}$ and $+0^{\circ}$ in declination. For the LHS stars without Durchmusterung numbers, finding charts are available (Luyten \& Albers 1979). The NLTT stars could be identified due to their large proper motion by cross-matching DSS1 with DSS2 which provide in this zone an epoch difference of the order of 15 years. The fourteen stars that could be successfully observed are listed in Table 1 together with the red and photographic magnitudes, spectral classes, and annual proper motions as published by
Table 1. NLTT values of the observed stars

\begin{tabular}{lcclcc}
\hline name & $m_{R}$ & $m_{\mathrm{pg}}$ & $\mathrm{Sp}$ & $\begin{array}{c}\mu \\
{\left[^{\prime \prime} / \mathrm{yr}\right]}\end{array}$ & $\theta$ \\
\hline LHS 2112 & 15.7 & 18.4 & $\mathrm{~m}+$ & 0.884 & $225^{\circ}$ \\
LP 609-31 & 15.3 & 17.4 & $\mathrm{~m}+$ & 0.356 & 254 \\
LP 609-47* & 16.4 & 19.0 & $\mathrm{~m}+$ & 0.407 & 131 \\
G 53-34 & 13.2 & 15.0 & $\mathrm{~m}$ & 0.407 & 131 \\
LP 609-52 & 16.9 & 19.0 & $\mathrm{~m}+$ & 0.450 & 137 \\
LP 790-15 & 16.8 & 18.1 & $\mathrm{k}-\mathrm{m}$ & 0.395 & 126 \\
LP 790-28 & 16.6 & 18.4 & $\mathrm{~m}$ & 0.407 & 269 \\
LP 731-16 & 13.6 & 16.0 & $\mathrm{~m}+$ & 0.486 & 272 \\
LP 672-8 & 15.7 & 17.7 & $\mathrm{~m}$ & 0.357 & 261 \\
LP 672-12 & 15.5 & 18.0 & $\mathrm{~m}+$ & 0.354 & 259 \\
LP 732-15 & 13.3 & 15.5 & $\mathrm{~m}+$ & 0.408 & 250 \\
LHS 334 & 16.9 & 19.7 & $\mathrm{~m}+$ & 1.338 & 168 \\
LP 796-12 & 16.2 & 18.4 & $\mathrm{~m}+$ & 0.438 & 239 \\
LHS 2662 & 17.1 & 18.7 & $\mathrm{~m}$ & 0.516 & 322 \\
\hline
\end{tabular}

* Common p. m. to G 53-34, separation $15^{\prime \prime} 5$ in $274^{\circ}$.

Luyten and supplied by the digitized version (Warren et al. 1989) of the NLTT catalogue.

For all red (Luyten's spectral classes " $\mathrm{m}$ " and " $\mathrm{m}+$ ") NLTT stars in the appropriate sky region, new photometry ( $R$ and $B_{j}$ magnitudes) and precise positions were obtained with the APM sky catalogues (Irwin et al. 1994, http://www.ast.cam.ac.uk/ ${ }^{\sim}$ apmcat/). A discussion of the APM magnitudes and their relation to Luyten's estimates can be found in Scholz et al. (2000). In the same paper, a rough correlation between spectral type and the APM measured $B_{j}-R$ colour is given. This colour was a secondary criterion for the selection of our candidate stars. The NLTT stars measured on UKST plates should have a $B_{j}-R \geq+2.0$. Several LHS stars lacking spectral types were added to the observing list. These were selected among faint candidates in the ARI data base of nearby stars (see e.g. Jahreiß \& Wielen 1997). Contrary to the NLTT stars, these LHS stars north of declination $+20^{\circ}$ were measured on POSS-I plates and $O-E \geq+3.5$ was chosen as lower limit.

Low-resolution spectra of the NLTT and LHS stars were obtained in spring 1999 during two observing runs at the German-Spanish Astronomical Centre on Calar Alto, Spain. While the aim of the observing programs was, in fact, spectroscopic follow-up observations of quasar candidates, relatively bright $\left(R<17^{\mathrm{m}}\right)$ proper motion stars are ideal targets during the twilight and during periods of weather conditions that are too bad for faint-object $\left(B>19^{\mathrm{m}}\right)$ spectroscopy.

Nine high proper motion stars have been observed with CAFOS, the focal reducer and faint object spectrograph at the $2.2 \mathrm{~m}$ telescope. CAFOS was equipped with the grism B-400 and a SITe1d CCD yielding $9.8 \AA$ per pixel and a wavelength coverage from 4000 to $8000 \AA$. A slit width of $1^{\prime \prime} .0$ or $1^{\prime \prime} .2$ was used, corresponding to a spectral resolution of 18 or $22 \AA$, respectively. Exposure times range from a few seconds for the brightest objects up to $300 \mathrm{~s}$ for the fainter ones. 
Table 2. APM positions and photometry, spectral types, distances and tangential velocities

\begin{tabular}{|c|c|c|c|c|c|c|c|c|c|c|c|c|}
\hline \multirow{2}{*}{$\begin{array}{c}\text { name } \\
\text { LHS } 2112\end{array}$} & \multicolumn{2}{|c|}{ right ascension } & $\begin{array}{l}\text { sion } \\
\text { J20 }\end{array}$ & \multicolumn{3}{|c|}{ declination } & \multirow{2}{*}{$\begin{array}{l}\text { epoch } \\
-1900 \\
54.179\end{array}$} & $R$ & $B_{j}-R$ & \multirow{2}{*}{ 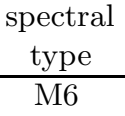 } & \multirow{2}{*}{$\begin{array}{c}\begin{array}{c}d \\
{[\mathrm{pc}]}\end{array} \\
21\end{array}$} & \multirow{2}{*}{$\frac{\left[\mathrm{km} \mathrm{s}^{-1}\right]}{87}$} \\
\hline & $09^{\mathrm{h}}$ & $09^{\mathrm{m}}$ & $28^{\mathrm{s}} .79$ & $+49^{\circ}$ & $39^{\prime}$ & $58^{\prime \prime} .5$ & & $16.2^{\mathrm{a}}$ & $+3.8^{b}$ & & & \\
\hline LP 609-31 & 10 & 06 & 04.91 & -01 & 46 & 24.7 & 86.026 & 15.8 & +2.2 & M3 & 159 & 271 \\
\hline LP 609-47 & 10 & 13 & 42.87 & +00 & 00 & 44.2 & 87.025 & 16.8 & +2.2 & M6 & 28 & 54 \\
\hline G 53-34 & 10 & 13 & 43.92 & +00 & 00 & 43.1 & 87.025 & 13.4 & +2.0 & M3.5 & 32 & 62 \\
\hline LP 609-52 & 10 & 15 & 21.05 & -01 & 53 & 30.7 & 87.025 & 16.9 & +2.0 & $\mathrm{M} 3.5^{\mathrm{c}}$ & 158 & 337 \\
\hline LP 790-15 & 10 & 24 & 55.92 & -17 & 46 & 24.2 & 88.207 & 16.1 & +2.2 & sdM1.5 & 150 & 285 \\
\hline LP 790-28 & 10 & 37 & 03.04 & -18 & 27 & 44.3 & 93.087 & 15.9 & +2.2 & M4 & 73 & 142 \\
\hline LP 731-16 & 10 & 53 & 02.50 & -15 & 05 & 03.5 & 89.324 & 12.6 & +2.2 & M3.5 & 22 & 51 \\
\hline LP $672-8$ & 11 & 11 & 31.23 & -06 & 10 & 27.6 & 85.057 & 15.9 & +2.1 & $\operatorname{sdM}^{\mathrm{c}}$ & & \\
\hline LP $672-12$ & 11 & 15 & 02.95 & -04 & 15 & 40.9 & 86.203 & 15.9 & +2.0 & M3.5 & 100 & 166 \\
\hline LP 732-15 & 11 & 17 & 06.79 & -11 & 22 & 23.4 & 87.083 & 11.8 & +2.6 & M3 & 25 & 49 \\
\hline LHS 334 & 12 & 34 & 14.77 & +20 & 38 & 04.4 & 55.387 & $16.8^{\mathrm{a}}$ & $+4.1^{\mathrm{b}}$ & $\mathrm{sdM} 4.5$ & 74 & 472 \\
\hline LP 796-12 & 12 & 51 & 55.50 & -16 & 14 & 09.1 & 88.281 & 15.7 & +1.9 & M6 & 17 & 35 \\
\hline LHS 2662 & 13 & 00 & 08.55 & +30 & 38 & 39.4 & 55.288 & $16.1^{\mathrm{a}}$ & $+3.9^{\mathrm{b}}$ & M6 & 20 & 49 \\
\hline
\end{tabular}

${ }^{a} E$ magnitude measured on POSS-I plate.

b $O-E$ colour measured on POSS-I plates.

c Spectrum taken with very low signal-to-noise ratio.

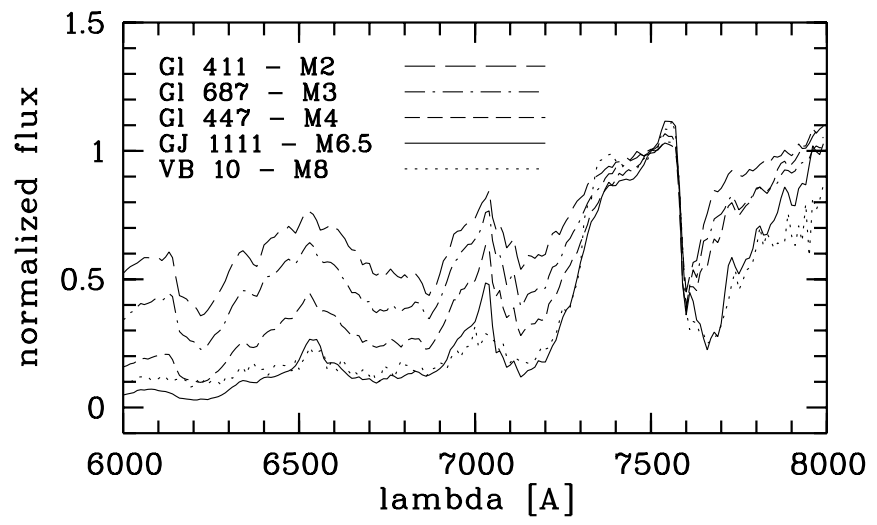

Fig. 1. The comparison spectra $\left(F_{\lambda}\right.$ normalized at $\left.7500 \AA\right)$ taken at Calar Alto of well-known nearby $\mathrm{M}$ dwarfs from top to bottom: M2 (Gl 411) to M8 (vB $10=$ Gl 752B). The differences between the earlier types are due to strengthening TiO bands. For the latest types, the $\mathrm{TiO}$ bandstrengths largely saturate, so the main difference seen is in the redness of the spectrum, the height of the pseudo-continuum peaks near 6500 and $7000 \AA$, and the onset of VO absorption near 7400 and $8000 \mathrm{~A}$

Low-resolution spectra for six objects were taken with the $3.5 \mathrm{~m}$ telescope on May 1999. The MOSCA instrument was used in single slit mode with the g250 grating, which has 250 rulings $\mathrm{mm}^{-1}$ and a blaze wavelength of $5700 \AA$. The spectra cover a wavelength range from $4000 \AA$ to $8000 \AA$. Using a $1.5^{\prime \prime}$ wide slit, the spectral resolution is about $20 \AA$. The exposure times range from twenty seconds to one minute.

The spectra were calibrated with the MIDAS package involving standard procedures for bias-subtraction, flat-fielding and wavelength calibration using Ne-Ar lamp spectra. For relative flux calibration we have used spectra of secondary standard stars for spectrophotometry from Oke \& Gunn (1983).
The atmospheric correction function for broad molecular absorption was derived from the standard stars to correct the atmospheric absorption in all spectra. As seen in Figs. 2 and 3, there seem to be systematic errors of the atmospheric correction around the $\mathrm{O} 2$ band at $7600 \AA$ for the $3.5 \mathrm{~m}$-spectra due to bad weather and varying sky conditions for the targets as well as for the standard stars. However, these errors did not influence the spectral classification.

Spectral types were estimated from comparing the spectra with a sequence of standard spectra of known M stars. This sequence of standard spectra was simultaneously taken during the observations carried out at Calar Alto and is presented in Fig. 1.

The spectral classification of the subdwarfs was carried out by a determination of the spectroscopic indices TiO5, $\mathrm{CaH} 1, \mathrm{CaH} 2, \mathrm{CaH} 3$, and a subsequent application of the precepts developed in Gizis (1997).

\section{Results}

The APM measurements of positions and magnitudes, as well as the spectral type determined from spectra taken at Calar Alto, and the resulting distances and tangential velocities are summarized in Table 2. In Col. 1 the designation of the star is given. Columns 2 and 3 list right ascension and declination for equinox J2000.0, and the plate epoch is found in Col. 4. The mean error in one coordinate amounts to \pm 0.2 arcsec for the NLTT stars and \pm 0.3 arcsec for the LHS stars. All APM catalogue J2000 astrometry is now based on Tycho-2 (Hœe et al. 2000).

The $R$ magnitude ( $E$ magnitude on POSS-I plates for the LHS stars) measured by the APM facility on UKST plates, are given in Col. 5, whereas Col. 6 lists the corresponding $B_{j}-R$ colour index which should have an 


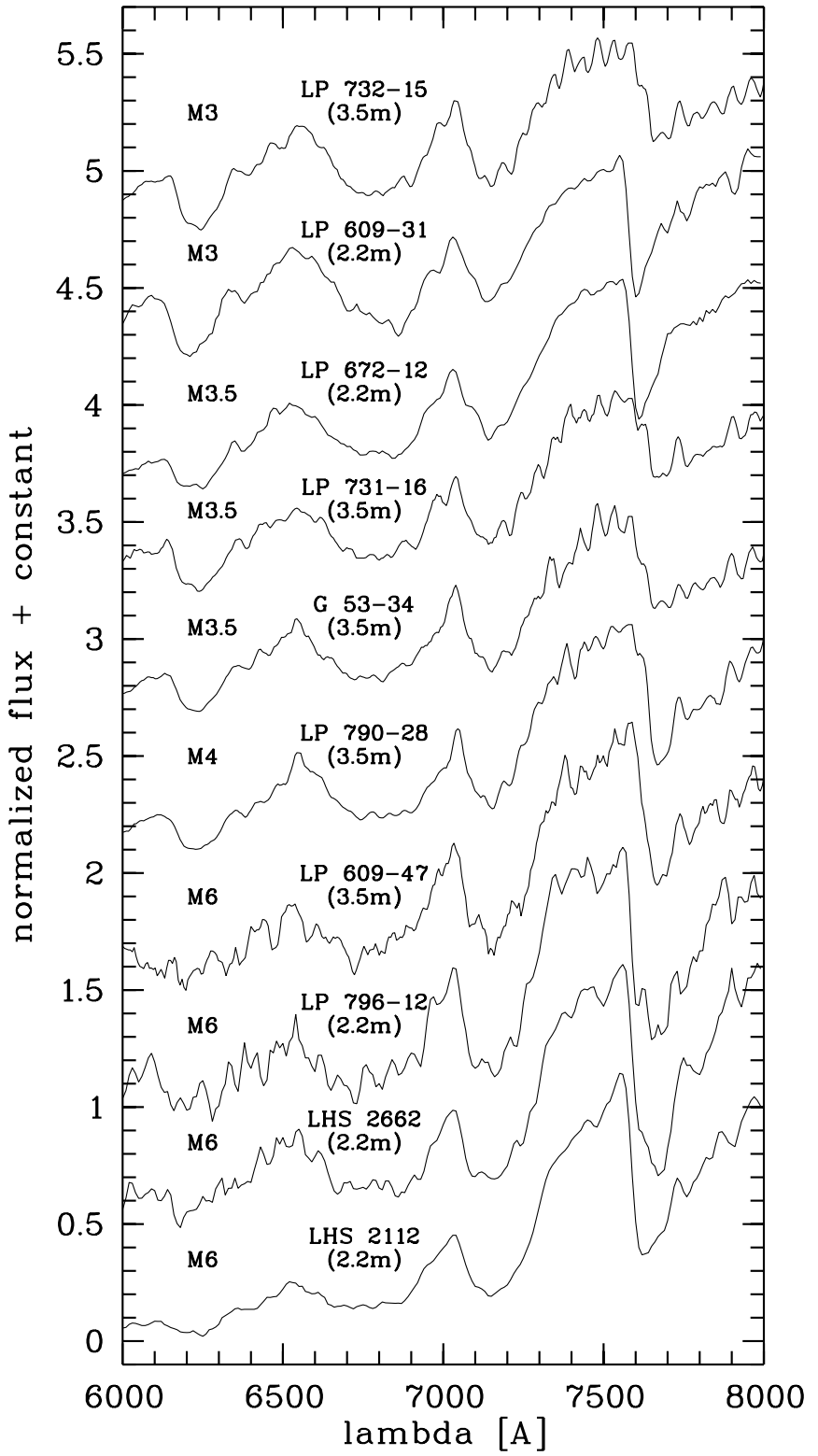

Fig. 2. Spectra of M dwarfs with spectral types from M3 (top) to M6 (bottom). The spectra $\left(F_{\lambda}\right)$ were normalized at $7500 \AA$ and shifted by a constant. The telescope used is given in brackets. The $2.2 \mathrm{~m}$-spectra were smoothed with a running mean filter over three pixels. For the more noisy spectra from the $3.5 \mathrm{~m}$ telescope taken by even worse weather conditions we applied a running mean over 5 pixels

internal accuracy of \pm 0.2 magnitudes (Scholz et al. 2000) (again $O-E$ was measured for the LHS stars).

The spectral type is listed in Col. 7. Two of the spectra were taken with very low signal-to-noise ratio, so that for the first object LP 609-52 only a very crude subtype and for the second object LP 672-8 even no subtype could be given. From the spectral type of the $\mathrm{M}$ dwarfs, an absolute magnitude $M_{R}$ was determined according to the calibration presented in Kirkpatrick \& McCarthy (1994). The distance modulus then followed from a comparison of this absolute magnitude with the APM measured $R$ magnitude, and the resulting distance is listed in Col. 8. The

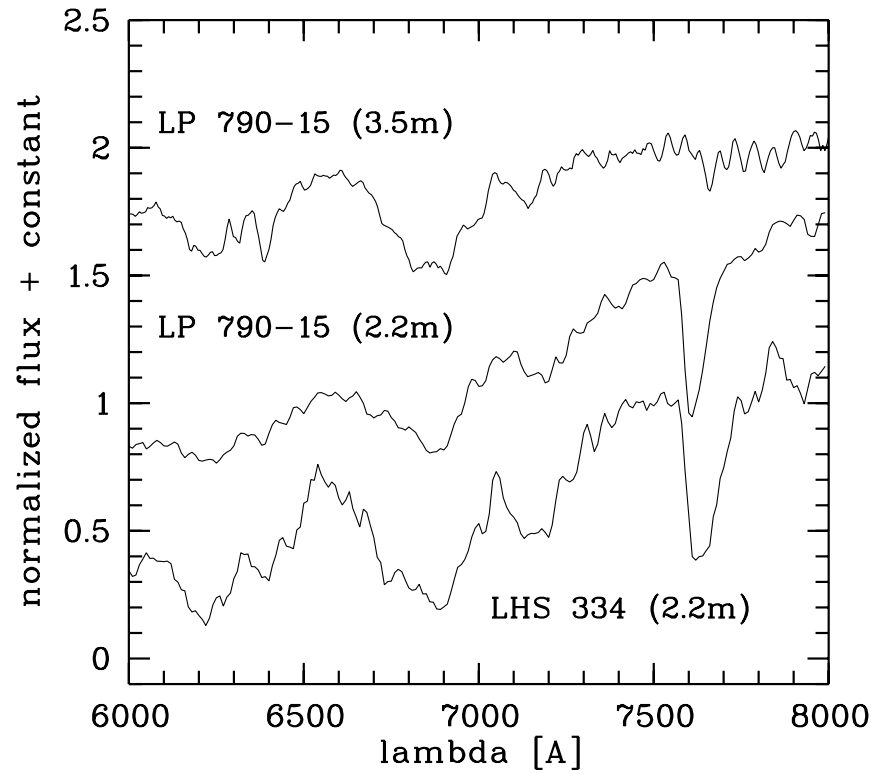

Fig. 3. Spectra of two subdwarfs $\left(F_{\lambda}\right.$ normalized at $7500 \AA$ and shifted by a constant). Although the spectra of LP 790-15, taken with the $3.5 \mathrm{~m}$ and $2.2 \mathrm{~m}$ telescopes, look somewhat different, we obtained very similar spectral indices classifying LP 790-15 as sdM1.5 in the system of Gizis (1997). For LHS 334, a late subdwarf classification sdM4.5 was obtained

distances derived for the two subdwarfs were deduced from the absolute magnitudes of similar stars in the compilation of Gizis (1997). Finally, in Col. 9 the resulting tangential velocities are given allowing a guess as to which age component of our Milky Way the star may belong.

In Fig. 2 the $\mathrm{M}$ dwarf spectra are shown. The spectra $\left(F_{\lambda}\right)$ were normalized at $7500 \AA$ and integral offsets added to the normalized flux to separate the spectra vertically. The spectra are arranged from top (M3) to bottom (M6) according to their subtype. The telescope used is given in brackets. The $2.2 \mathrm{~m}$-spectra were smoothed with a running mean filter over three pixels. The spectra from the $3.5 \mathrm{~m}$ telescope were taken during even worse weather conditions and are more noisy. For these, a running mean over five pixels was applied.

The subdwarf spectra are shown in Fig. 3. Again the spectra $\left(F_{\lambda}\right)$ were normalized at $7500 \AA$ and shifted vertically by a constant, and the same smoothing procedures as for the $\mathrm{M}$ dwarf spectra were applied. A spectrum for LP 790-15 was taken by both telescopes. Despite the fact that the two spectra (see top of Fig. 3) look somewhat different, very similar spectral indices were obtained resulting in a sdM1.5 classification. At bottom of Fig. 3 the sdM4.5 spectrum of LHS 334 is shown. The subdwarf character of this very high proper motion star rules out that it belongs to the immediate solar neighbourhood. Not plotted were the spectra of the M subdwarf LP 672-8 and the M3.5 dwarf LP 609-52. Both were obtained with the $2.2 \mathrm{~m}$ telescope under a very low signal-to-noise ratio.

Eleven of our stars could be identified in the 2 Micron All Sky Survey (2MASS) Point Source Catalogs (Second 
Incremental Release). This gave us the opportunity to obtain for these objects more recent positions as well as more precise photometry in the $J, H$, and $K_{\mathrm{s}}$ band, and, along with the calibration in Kirkpatrick \& McCarthy (1994), independent spectrophotometric distance estimates. The 2MASS data are presented in Table 3 in a form analogous to the APM data in Table 2. We see that the resulting distances (Col. 9) are in good agreement with the former ones, except for the M6 dwarf LP 796-12 whose 2MASS distance becomes considerably larger. In view of the fact that for all other stars in our sample red and infrared distances agree rather well, one may speculate that LP 796-12 consists of an unresolved red dwarf-white dwarf pair.

Among the fourteen stars investigated, there are four M6 dwarfs, presumably all within about $30 \mathrm{pc}$ of the Sun. From the remaining seven M3 to M4 dwarfs, four seem to be much further away. This applies also to the three subdwarfs in our sample.

\section{Individual stars}

LHS 2112 = LP 165-29: A trigonometric parallax of $39.1 \pm 6.4$ mas was published for this star in the Yale Parallax Catalog (van Altena et al. 1995). Parallax and photometry $\left(V=17^{\mathrm{m}} .46\right.$ and $\left.B-V=+1.79\right)$ originate from the still unpublished 10th parallax list of the US Naval Observatory. The unusual large error of this USNO parallax may indicate that it is only a preliminary value. Monet \& Dahn (1983) list LHS 2112 in an early paper on CCD parallaxes among the stars in fields awaiting further observations adding a remark denotes marginally acceptable field which may later prove unacceptable. So, our smaller spectroscopic distance estimate as implied from its dwarf M6 spectral type is not too implausible. A spectral type of M4: V is listed in Kirkpatrick (1992) coming from a private 1992 communication from Pat Boeshaar.

G 53-34 and LP 609-47: For G 53-34 Ed Weis (1986) measured $V R I$ photometry $V=14.89, V-R=1.43$, and $R-I=1.23$ ( $R I$ in the Kron-system). From these values he deduced a photometric parallax of 31 mas.

Though he was aware of the common proper-motion companion LP 609-47 detected by Luyten he made no photometric observations of it because LP 609-47 was far below the magnitude limit $m_{R} \leq 13 .{ }^{m} 5$ of his photometric survey. Spectroscopic and photometric distance estimates for the brighter component are in good agreement, and also the spectroscopic distance for the fainter component fits fairly well.

LP 609-52: The very low signal-to-noise ratio of its spectrum permitted only a coarse $( \pm 1.5$ subclasses $)$ determination of the spectral type. Therefore, also distance and tangential velocity remain rather uncertain. In any case, its distance should be far beyond $25 \mathrm{pc}$.

LP 790-15: From the spectral indices $\mathrm{TiO} 5=0.85$, $\mathrm{CaH} 1=0.75, \mathrm{CaH} 2=0.50$, and $\mathrm{CaH} 3=0.85$, it can be deduced that LP 790-15 is an early M-type subdwarf. The classification system of Gizis (1997) yields sdM1.5 as subtype. Therefore, it should be at a distance of about $150 \mathrm{pc}$.

LP 672-8: The spectrum of this star shows probably an M subdwarf. However, the very low signal-to-noise ratio prevented us from measuring the spectroscopic indices required for a determination of the subtype. Even as a late sdM5.0 subdwarf, its distance would be already about $40 \mathrm{pc}$.

LP 732-15: This is the brightest star in our sample, and it is identical with WT 1836, i.e. it was rediscovered as high proper motion star by Wroblewski \& Torres (1997). These authors measured a photographic magnitude of 15.5 for it. Nevertheless, apart from our spectral type M3, indicating a distance of $25 \mathrm{pc}$, no additional informations seem to be published for this relatively bright star.

LHS 334 = GJ 2093: This star was included in the list of Suspected Nearby Stars (Table 2 in Gliese \& Jahreiß 1979) merely due to its faintness, Luyten's spectral class "m+", and the very high proper motion without any further information. In their paper on preliminary CCD parallaxes, Monet \& Dahn (1983) list LHS 334 among the stars with unacceptable reference frames.

With $O-E=+4.1$, LHS 334 belongs to the reddest stars in our sample, and it is at the same time the star with largest proper motion (1.34 arcsec/yr). However, the present spectrum (see the bottom panel of Fig. 3) indicates that it is a late M-type subdwarf.

The spectroscopic indices $\mathrm{TiO} 5=0.6, \mathrm{CaH} 1=0.6$, $\mathrm{CaH} 2=0.3$, and $\mathrm{CaH} 3=0.6$ yield a spectral type sdM4.5, according to the spectral classification proposed by Gizis (1997). This is quite close to the spectral type of sdM4 listed in Kirkpatrick (1992) according to a private 1992 communication from Pat Boeshaar. From our spectral type a distance of almost $74 \mathrm{pc}$ and a tangential velocity of more than $470 \mathrm{~km} \mathrm{~s}^{-1}$ can be assumed for LHS 334 .

LP 796-12 was found to be the nearest star in our sample according to the distance modulus calculated from the APM measured $R$-magnitude. Though its color index $B_{j}-R=+1.9$ is rather small, the spectral type (Fig. 2) is M6 and the corresponding distance would be $17 \mathrm{pc}$. Applying the infrared magnitudes provided by 2MASS yields a considerably larger distance of almost 26 pc. Such a discrepancy can occur, for example, if LP 796-12 is an unresolved $\mathrm{dM}+\mathrm{DA}$ pair. To our knowledge this is the first distance estimate for LP 798-12.

LHS 2662 = LP 322-288 (in SIMBAD to be found also as separate entry [SIP89] 125744.3+305458): Stobie et al. (1990) obtained a first distance estimate for this star. Their absolute magnitude calibration in combination with the measured photographic photometry $V=18{ }^{\mathrm{m}} 01$, $V-I=3.97$ ( $V-I$ in the Kron-Cousins system) yields an absolute visual magnitude $M_{V}=16$. 15 corresponding to a distance of $24 \mathrm{pc}$. From this estimate the star entered the CNS (Catalogue of Nearby Stars) data base. Then it was removed from the preliminary version of CNS3 (Gliese \& Jahreiß 1991) due to a decision to take photographic photometry out of consideration. 
Table 3. 2MASS positions and photometry, spectral types, distances and tangential velocities

\begin{tabular}{|c|c|c|c|c|c|c|c|c|c|c|c|c|c|}
\hline \multirow{2}{*}{$\begin{array}{c}\text { name } \\
\text { LHS } 2112\end{array}$} & & t asce & $\begin{array}{l}\text { sion } \\
\text { J20 }\end{array}$ & \multicolumn{3}{|c|}{ declination } & $\begin{array}{l}\text { epoch } \\
-1900 \\
\end{array}$ & $J$ & $H$ & $\overline{K_{\mathrm{s}}}$ & \multirow{2}{*}{$\begin{array}{c}\text { spectral } \\
\text { type }\end{array}$} & $\begin{array}{c}d \\
{[\mathrm{pc}]}\end{array}$ & $\begin{array}{r}v_{\mathrm{t}} \\
{\left[\mathrm{km} \mathrm{s}^{-1}\right]}\end{array}$ \\
\hline & $09^{\mathrm{h}}$ & $09^{\mathrm{m}}$ & $25^{\mathrm{s}} .92$ & $+49^{\circ}$ & $39^{\prime}$ & $29^{\prime \prime} 6$ & 99.178 & 11.717 & 11.141 & 10.793 & & 20 & 84 \\
\hline LP 609-31 & 10 & 06 & 04.63 & -01 & 46 & 26.0 & 99.140 & 12.826 & 12.339 & 12.041 & M3 & 142 & 240 \\
\hline LP 609-47 & 10 & 13 & 43.14 & +00 & 00 & 41.0 & 98.989 & 12.530 & 11.944 & 11.578 & M6 & 29 & 56 \\
\hline G 53-34 & 10 & 13 & 44.19 & +00 & 00 & 39.9 & 98.989 & 10.599 & 10.011 & 9.697 & M3.5 & 34 & 66 \\
\hline LP 609-52 & 10 & 15 & 21.29 & -01 & 53 & 34.5 & 98.989 & 13.292 & 12.747 & 12.536 & $\mathrm{M} 3.5^{\mathrm{c}}$ & 119 & 254 \\
\hline LP 790-15 & 10 & 24 & 56.20 & -17 & 46 & 26.6 & 99.099 & 13.905 & 13.396 & 13.155 & sdM1.5 & 158 & 296 \\
\hline LP 731-16 & 10 & 53 & 02.22 & -15 & 05 & 03.3 & 98.293 & 9.899 & 9.360 & 9.020 & M3.5 & 25 & 58 \\
\hline LP $672-8$ & 11 & 11 & 30.91 & -06 & 10 & 28.2 & 99.101 & 12.457 & 11.901 & 11.621 & $\mathrm{sdM}^{\mathrm{c}}$ & & \\
\hline LP $672-12$ & 11 & 15 & 02.66 & -04 & 15 & 41.2 & 99.011 & 12.829 & 12.232 & 11.944 & M3.5 & 95 & 159 \\
\hline LP 796-12 & 12 & 51 & 55.27 & -16 & 14 & 11.4 & 98.244 & 12.249 & 11.698 & 11.430 & M6 & 26 & 54 \\
\hline LHS 2662 & 13 & 00 & 07.47 & +30 & 38 & 55.9 & 98.194 & 11.904 & 11.301 & 11.021 & M6 & 22 & 54 \\
\hline
\end{tabular}

${ }^{\mathrm{c}}$ Spectrum taken with very low signal-to-noise ratio.

Reid (1990) reported similar photometric values: $V=$ 18. 03 and $V-I=4.16$. The discrepancy in $V-I$ may be attributed to different photographic emulsion/filter combinations. The infrared photometry $K=10$ m $95, J-K=$ 0.95 , and $H-K=0.37$ also measured by Stobie et al. (1990) is in excellent agreement with other M6 dwarfs (see Table 3 in Kirkpatrick \& McCarthy 1994).

\section{Conclusions}

Broad wavelength spectroscopy was carried out for a list of fourteen red high proper motion stars selected from NLTT or LHS, and mostly without any previous photometric or spectroscopic information. The present results demonstrate that such observations within the scope of a spectroscopic back-up programme were able to provide within a short time interval valuable information on presumably nearby stars. In a preparatory step APM measurements supplied precise coordinates and improved color estimates with respect to the ones given in the NLTT catalogue. Nevertheless, looking at our results, there seems to be only a rather weak correlation between the $B_{j}-R$ colour index and the final spectral type. From the present data in Tables 2 and 3 it becomes evident that a better correlation can be obtained by taking instead the $R-K_{\mathrm{S}}$ colour index.

It can be recognized that eleven of the stars investigated were classified as $\mathrm{M}$ dwarfs with subtypes ranging from M3 to M6. Among them are four M6 stars. Three stars (LP 790-15, LP 672-8, and LHS 334) turned out to be subdwarfs of type M.

Spectrophotometric distances were determined from the APM measured $R$-magnitudes and for most stars also from infrared magnitudes found in the 2MASS Point Source Catalog. Both distance estimates agree rather well, except for LP 796-12, where the much larger distance from the infrared magnitudes may indicate the presence of a fainter white dwarf companion. For three $M$ dwarfs our spectroscopic distance estimates are in satisfactory agreement with independent distances found in the literature.
Neglecting the possibility that the one or other star in our sample may consist of an unresolved binary, our spectroscopic distances show about 40 per cent of the observed stars to be nearby, i.e. within 25 pc. In total, about 50 per cent of the present star sample should be promising targets for a trigonometric parallax programme. For numerous additional high-proper motion stars spectra have been acquired and will be investigated and presented in a forthcoming paper.

The present targets were selected according to their high proper motion. This allows a unique identification on APM plates by comparing plates taken at different epochs. A subsequent selection of red $B_{j}-R$ colours should have improved the probability to concentrate on late type dwarf stars. This strategy has the disadvantage to restrict the search mainly on the high velocity tail of the local M dwarf distribution.

Now to a large extend infrared magnitudes have become availabe in the form of the 2MASS and DENIS surveys. A pairing of these infrared data bases with digitized sky surveys provides at the same time optical and infrared colours and by their combination, e.g. $R-K_{\mathrm{s}}$, much improved selection criteria (Kirkpatrick et al. 1998; Forveille et al. 1998). In addition, and even more important, such a search can easily be extended to the low velocity part of the local M dwarf distribution and thereby help to remove the kinematical bias still present in the sample of faint nearby stars.

Acknowledgements. We are indepted to the referee, J. Davy Kirkpatrick, for his very detailed and useful comments, especially for his suggestion to incorporate the 2MASS data that resulted in a considerable improvement of the paper.

This research has made use of the SIMBAD database, Strasbourg, of the STScI Digitized Sky Survey, particularly of the batch service of the National Astronomical Observatory of Japan and of data products from the Two Micron All Sky Survey, which is a joint project of the University of Massachusetts and the Infrared Processing and Analysis Center, funded by the National Aeronautics and Space Administration and the National Science Foundation. 
RDS gratefully acknowledges financial support from the Deutsches Zentrum für Luft- und Raumfahrt (DLR) (Förderkennzeichen 01 OI 98017 and 50 OI 0001).

\section{References}

Argelander, F. W. 1859-62, Bonner Durchmusterung nördlicher Teil. Astron. Beob. Bonn 3 - 5, 3rd edition Dümmler Verlag, Bonn, 1951

Eggen, O. J. 1979, ApJS, 39, 89

Eggen, O. J. 1980, ApJS, 43, 457

Eggen, O. J. 1987, AJ, 92, 379

Forveille, T., Delfosse, X., \& Epchtein, N. 1998, in The Impact of Near-Infrared Sky Surveys on Galactic and Extragalactic Astronomy, ed. N. Epchtein, Astrophys. Space Sci. Libr., 230,101

Gizis, J. E. 1997, AJ, 113, 806

Gliese, W., \& Jahreiß, H. 1979, A\&AS, 38, 423

Gliese, W., \& Jahreiß, H. 1991, Preliminary Version of the Third Catalogue of Nearby Stars, in The Astronomical Data Center CD-ROM, Selected Astronomical Catalogs, vol. I, ed. L. E. Brotzman, \& S. E. Gessner

Henry, T. J., Kirkpatrick, J. D., \& Simons, D. A. 1994, AJ, 108,1437

Hog, E., Fabricius, C., Makarov, V. V., et al. 2000, A\&A, 355, L27

Ibata, R., Irwin, M., Bienayme, O., Scholz, R., \& Guibert, J. 2000, ApJ, 532, L41

Jahreiß, H., \& Wielen, R. 1997, in Hipparcos Venice '97, ed. M. A. C. Perryman, \& P. L. Bernacca, ESA SP-401, 675

Kirkpatrick, J. D. 1992, Ph.D. Thesis, Univ. of Arizona

Kirkpatrick, J. D. 1995, in The Bottom of the Main Sequence - and beyond, ed. C. G. Tinney, Proc. ESO Workshop (Springer-Verlag Berlin Heidelberg New York), 140

Kirkpatrick, J. D., \& McCarthy, D. W. 1994, AJ, 107, 333
Kirkpatrick, J. D., Skrutskie, M. F., Liebert, J., et al. 1998, in The Impact of Near-Infrared Sky Surveys on Galactic and Extragalactic Astronomy, ed. N. Epchtein, Astrophys. Space Sci. Libr., 230, 109

Luyten, W. J. 1979-1980, New Luyten Catalogue of Stars with Proper Motions Larger than Two Tenths of an Arcsecond, Univ. of Minnesota, Minneapolis, vols. 1-4.

Luyten, W. J., \& Albers, H. 1979, An Atlas of Identification Charts for LHS Stars, Univ. of Minnesota, Minneapolis

Irwin, M. J., Maddox, S. J., \& McMahon, R. 1994, Spectrum No. 2, p. 14

Monet, D. G., \& Dahn, C. C. 1983, AJ, 88, 1489

Oke, J. B., \& Gunn, J. E. 1983, ApJ, 266, 713

Reid, N. 1990, MNRAS, 247, 70

Schönfeld, E. 1886, Bonner Durchmusterung südlicher Teil. Astron. Beob. Bonn 8, 2nd edition Dümmler Verlag, Bonn, 1949

Scholz, R.-D., Irwin, M., Ibata, R., Jahreiß, H., \& Malkov, O.Yu. 2000, A\&A, 353, 958

Scholz, R.-D., Irwin, M., Schweitzer, A., \& Ibata, R. 1999, A\&A, 345, L55

Schweitzer, A., Scholz, R.-D., Stauffer, J., Irwin, M., \& McCaughrean, M. J. 1999, A\&A, 350, L62

Stobie, R. S., Ishida, K., \& Peacock, J. A. 1990, MNRAS, 238, 709

Thome, J., \& Perrine, C. D. 1892-1932, Cordoba Durchmusterung. Resultados del Observatorio National Argentino vols. $16-18,21$ parts I \& II

van Altena, W. F., Lee, J. T., \& Hoffleit, E. D. 1995, The General Catalogue of Trigonometric Stellar Parallaxes, Fourth Edition, Yale Univ. Obs., New Haven, USA

Warren, W. H. Jr., Sturch, C. R., Lasker, B. M., Jahreiß, H., \& Luyten, W. J. 1989, AJ, 97, 1480

Weis, E. W. 1986, AJ, 91, 626

Weis, E. W. 1986, AJ, 96, 1710

Weis, E. W. 1996, AJ, 112, 2300

Wroblewski, H., \& Torres, C. 1997, A\&AS, 122, 447 